\title{
EFFECTS OF COMPTON COOLING ON OUTFLOWS IN A TWO COMPONENT ACCRETION FLOW AROUND A BLACK HOLE: RESULTS OF A COUPLED MONTE CARLO-TVD SIMULATION
}

\author{
SUDIP K. GARAIN ${ }^{1, *}$, HIMADRI GHOSH ${ }^{2}$ and SANDIP K. CHAKRABARTI ${ }^{1,2}$ \\ 1 S.N. Bose National Centre for Basic Sciences, JD Block, Salt Lake, Sector III, Kolkata, \\ 700098, India \\ 2 Indian Centre for Space Physics, Chalantika 43, Garia Station Rd., Garia, Kolkata, \\ 700084 , India \\ *E-mail: sudip@bose.res.in
}

\begin{abstract}
The effect of cooling on the outflow rate from an accretion disk around a black hole is investigated using a coupled Monte Carlo Total Variation Diminishing code. A correlation between the spectral states and the outflow rates is found as a consequence.

Keywords: Accretion, accretion disks - Black hole physics - Hydrodynamics - Methods: numerical - Radiative transfer - Shock waves
\end{abstract}

\section{Introduction}

Outflows are common in many astrophysical objects which contain black holes. It is generally believed that the outflows originate from the inflowing gas itself. In a numerical simulation using total variation diminishing (TVD) code, Giri et al. (2010) showed that the outflow rates from an inviscid accretion flow strongly depend on the outward centrifugal force and upto $60 \%$ matter can be driven out of the disk. Here, we discuss how the Compton cooling affects the outflow from the accretion disk.

\section{Simulation Set Up and Procedure}

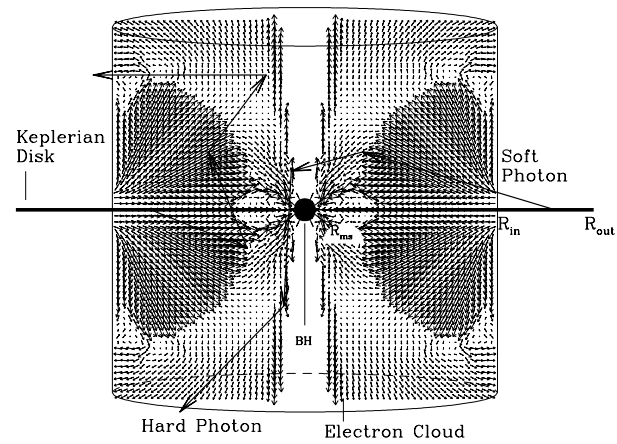

Fig. 1. Schematic diagram of the geometry of our radiative hydrodynamics simulations. Zigzag trajectories are the typical paths followed by the photons. The velocity vectors of the infalling matter inside the cloud are shown. The velocity vectors are plotted for $\lambda=1.73$.

In Figure 1, the schematic diagram of our simulation set up is presented. The outer boundary of the sub-Keplerian matter is at $R_{i n}=100 r_{g}$ whereas that of the 
Keplerian disk at the equatorial plane is located at $R_{\text {out }}=200 r_{g}$. At the center, a non-rotating black hole of mass $10 M_{\odot}$ is located. We have calculated the flow dynamics using the TVD code (Ryu et al. 1997; Giri et al. 2010). For a particular simulation, we use the Keplerian disk rate $\left(\dot{m}_{d}\right)$ and the sub-Keplerian halo rate $\left(\dot{m}_{h}\right)$ as parameters. The specific energy $(\epsilon)$ and the specific angular momentum $(\lambda)$ determines the hydrodynamics (shock location, number density and velocity variations etc.) and the thermal properties of the sub-Keplerian matter.

The radiative properties of the accretion disk is studied using a Monte Carlo code (Poznyakov et al. 1983; Ghosh et al. 2009). Each photon from the Keplerian disk is tracked till either it leaves the accretion disk or is absorbed by the black hole. If a scattering between an electron and a photon occurs on the way, the amount of energy exchanged is computed and suitably adjusted against the total energy of the electron and the photon.

The hydrodynamic code and the radiative transfer code is coupled together and are run back to back. In this way, we get an opportunity to see how the dynamics of the flow changes because of the Compton cooling and how the radiation spectrum from the accretion disk vary with time.

\section{Results and Discussions}

\begin{tabular}{cccc}
\multicolumn{4}{c}{ Table 1: Parameters used for the simulations. } \\
\hline Case & $\epsilon, \lambda$ & $\dot{m}_{h}$ & $\dot{m}_{d}$ \\
\hline Ia & $0.0021,1.76$ & 1.0 & No Disk \\
Ib & $0.0021,1.76$ & 1.0 & 0.5 \\
Ic & $0.0021,1.76$ & 1.0 & 1.0 \\
Id & $0.0021,1.76$ & 1.0 & 2.0 \\
\hline IIa & $0.0021,1.73$ & 1.0 & No Disk \\
IIb & $0.0021,1.73$ & 1.0 & 0.5 \\
IIc & $0.0021,1.73$ & 1.0 & 1.0 \\
IId & $0.0021,1.73$ & 1.0 & 2.0 \\
\hline
\end{tabular}

In Table 1, we list various Cases with all the simulation parameters used. In Cases Ia and IIa, there is no Keplerian disk. These are non-radiative hydro-dynamical simulations and no Compton cooing is included.

We find that the average temperature in the post-shock region is reduced rapidly as the disk rate $\dot{m}_{d}$ is increased keeping the halo rate $\dot{m}_{h}$ fixed for both the angular momenta cases. As a result, the average shock location decreases since cooling reduces the thermal pressure in this region. We now study how the Comptonization affects the outflow rates. We define the outflow rate $\dot{M}_{\text {out }}$ to be the rate at which outward pointing flow leaves the computational grid. In Figure 2, we plot the results of the time variation of the ratio $R_{\dot{m}}\left(\frac{\dot{M}_{\text {out }}}{\dot{M}_{i n}}\right), \dot{M}_{i n}$ being the constant injection rate on the right boundary. It can be clearly observed that with the increase in cooling the ratio is dramatically reduced and indeed becomes almost saturated as soon as some cooling is introduced.

The time variation of spectral properties of the disk is also studied. Whether the spectrum will be soft or hard is determined by the relative availability of the 

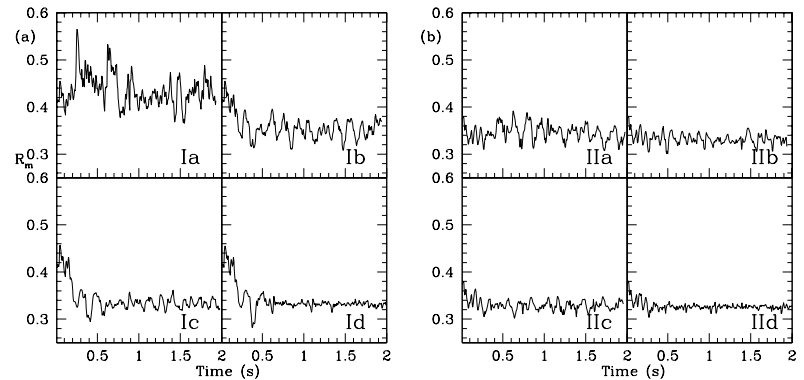

Fig. 2. Variations of $R_{\dot{m}}\left(=\frac{\dot{M}_{\text {out }}}{\dot{M}_{\text {in }}}\right)$ with time for different $\dot{m}_{d}$ is shown here. (a) $\lambda=1.76$ and (b) $\lambda=1.73$. The Cases are marked in each panel. The outflow rate is the lowest for the highest Keplerian disk accretion rate (Cases are Id and IId).

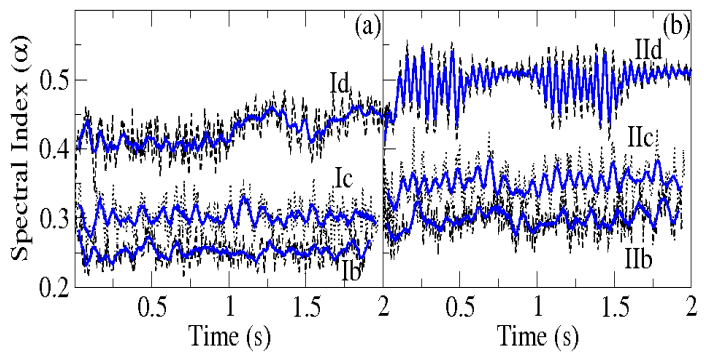

Fig. 3. Time variation of the spectral slope $\left(\alpha, I(E) \propto E^{-\alpha}\right)$ for different disk rates and their running averages (solid line) are shown. Different Cases are marked.

hot electrons and the soft photons in the disk and the jet. As the increase in $\dot{m}_{d}$ cools the post shock region and shrinks its size, the spectrum also becomes softer. If we define the energy spectral index $\alpha$ to be $I(E) \propto E^{-\alpha}$ in the region $1-10$ $\mathrm{keV}$, we note that $\alpha$ increases with the increase in $\dot{m}_{d}$. In Figure 3 , we present the time variation of the spectral index for the different Cases. We draw the running mean through these variations. We clearly see that the spectral index goes up with the increase in the disk accretion rate. Thus, on an average, the spectrum softens. We also find that the spectrum oscillates quasi-periodically and the frequency is higher for higher cooling rate. This agrees with the general observations that the quasi-periodic oscillation (QPO) frequency rises with luminosity (Cui et al. 1999; Sobczak et al. 2000; McClintock et al. 2009).

\section{References}

1. D. Ryu, S. K. Chakrabarti and D. Molteni, ApJ 474, 378 (1997)

2. K. Giri, S. K. Chakrabarti, M. M. Samanta, and D. Ryu, MNRAS 403, 516 (2010)

3. L. Poznyakov, I. Sobol, and R. Sunyaev, A \& Sp Physics Reviews 2, 189 (1983)

4. H. Ghosh, S. K. Chakrabarti and P. Laurent, IJMPD 18, 1693 (2009)

5. W. Cui, S. N. Zhang, W. Chen, et al. ApJ 512, L43 (1999)

6. J. E. McClintock, R. A. Remillard, M. P. Rupen, et al. ApJ 698, 1398 (2009)

7. G. J. Sobczak, J. E. McClintock, R. A. Remillard, et al. ApJ 531, 537 (2000) 